\title{
The Performances and Challenges of Today's EB Lithography and EB-resist Materials
}

\author{
Masato Saito, Kunihiro Ugajin, Keisuke Yagawa, Machiko Suenaga, and Yoshihito Kobayashi \\ Lithography Process Technology Dept., Center for Semiconductor Research \& Development \\ Toshiba Corp. Semiconductor \& Storage Product Company, \\ 1, Komukai-Toshiba-cho, Saiwai-ku, Kawaski, 212-8583, Japan \\ masato2.saito@toshiba.co.jp
}

\begin{abstract}
To investigate the possibility to catch up the NGL mask pattern size scaling strategy which indicated in ITRS2012, the performance of conventional mask fabricating process was examined. Current EB resist used for mask fabrication doesn't have enough performance to resolve below hp20nm pattern. With newly developed CAR resist, the resolution limit reached to hp18nm pattern. Furthermore by using higher performance EB writer, the possibility to resolve up to hp16nm pattern was showed. The impact of proximity effect to the resist performance was examined. The resist damage induced by proximity effect degrades the resolution limit about $2 \mathrm{~nm}$. This is a serious problem for NGL mask manufacturing. Reducing the impact of proximity effect is one of the major challenges for developing higher resolution EB resist.
\end{abstract}

Keywords: EB, mask, resist, proximity effect

\section{Introduction}

The EB lithography is still powerful and important tool in semiconductor manufacturing technology. Today, almost $100 \%$ of photomasks which used for advanced semiconductor device manufacturing are fabricated by using EB lithography technique. The EB lithography is supporting the foundation of semiconductor industry.

Aiming for minimizing semiconductor device's manufacturing costs, circuit patterns size of semiconductor devises have been shrinking. According to this trend, the pattern size on photomask has been shrinking too. Thoroughly, mask pattern's shrinking speed has been accelerated by the resolution enhancing technique like OPC and the next generation lithography (NGL) technology like EUV lithography.

Fig.1 shows the scaling roadmap of semiconductor device and mask indicated by ITRS2012 [1]. The circuit pattern size of semiconductor device shrinks to around $10 \mathrm{~nm}$ by 2020. And on the NGL mask, the pattern size shrinks according with device pattern size shrinkage. The pattern size on NGL mask goes below $20 \mathrm{~nm}$ in 2017, and reaches near $10 \mathrm{~nm}$ by 2020. But on the photomask, the pattern size shrinkage stops at around 40nm by 2016 because of the limits of light source's wavelength.

To catch up the scaling road map of NGL mask pattern size, the developments of the resolution enhancing technology on mask are needed. The researches for improving the performance of EB resist and EB writer has been executed for this objective.

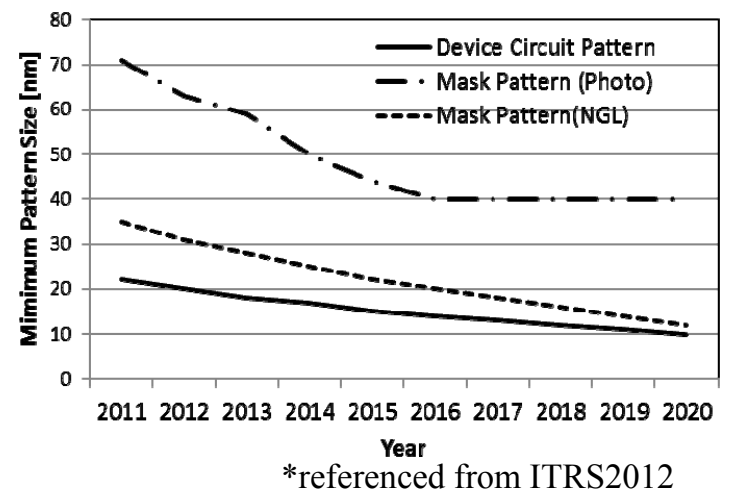

Fig.1. Pattern size scaling road map of semiconductor device and mask 


\section{Challenges for $E B$ resist}

\subsection{Target of resist sensitivity}

CAR (Chemical Amplified Resist) is used as resist material in mask manufacturing in general. Thanks to higher resist sensitivity of CAR, mask writing time becomes shorter, i.e. we can get higher productivity with CAR. To proceed pattern shrinking while maintain productivity is one of the major challenges on NGL mask manufacturing.

Writing a mask within a day is a guideline of the productivity of today's mask manufacturing. In today's common mask fabrication process, EB writer which has $50 \mathrm{kV}$ acceleration voltage and $\mathrm{EB}$ resists which has 10 to $30 \mathrm{uC} / \mathrm{cm}^{2}$ sensitivity on $50 \mathrm{kV}$ EB have been used. By these equipment and resist material, it takes more than 10 hours to finish writing a mask with today's common mask pattern data.

The mask writing time is estimated from shot counts and resist's sensitivity. The VSB type EB writer is mainly used for mask manufacturing. In VSB type EB writer, electron beam is shaped to rectangle or triangle figure, and each shaped beams are cyclically exposure on resist material according to the inputted mask pattern data. The number of shaped beams which exposed for one mask is called shot counts. The writing time of a mask is roughly estimated from the equation below [2],

$$
\mathrm{T}=\mathrm{N}\left(\frac{D}{J}+t_{s}\right)
$$

Here in,

$$
\begin{array}{ll}
\mathrm{T} & \text { : writing time } \\
\mathrm{N} & \text { : shot counts } \\
\mathrm{D} & \text { : exposure dosage for a shot } \\
\mathrm{J} & \text { : beam current density } \\
\mathrm{t}_{\mathrm{s}} & \text { : beam settling time }
\end{array}
$$

The exposure dosage " $\mathrm{D}$ " is decided from resist sensitivity, the beam current density "J" and the beam settling time " $\mathrm{s}_{\mathrm{s}}$ " are decided from EB writer's specification. And shot counts " $\mathrm{N}$ " is decided from the inputted mask pattern data.

Fig. 2 shows the trend of writing time of a mask which calculated from Equation (1). The shot counts " $\mathrm{N}$ " was estimated from the circuit design indicated in ITRS2012, and " $D$ ", " $\mathrm{J}$ " and " $\mathrm{t}_{\mathrm{s}}$ " were got from specification of conventional EB resist and EB writer. If the resist sensitivity won't be changed, the writing time of both photo and NGL mask will be below 24 hours by 2020 . But for eliminating pattern roughness caused by shot noise or process blur, the sensitivity of EB resist is getting lower these days.

Fig. 3 shows the estimation of NGL mask writing time depends on resist sensitivity. 3 different sensitivity, $30 \mu \mathrm{C} / \mathrm{cm}^{2}$ (conventional), $100 \mu \mathrm{C} / \mathrm{cm}^{2}$ and $300 \mu \mathrm{C} / \mathrm{cm}^{2}$ are used for estimation. The results on $300 \mu \mathrm{C} / \mathrm{cm}^{2}$ (10 times lower sensitivity than today's conventional one) is far from the guideline, it takes few days even in the today's mask pattern design. The results on $100 \mu$ $\mathrm{C} / \mathrm{cm}^{2}$ are more realistic than the former results, the writing time will be following near guideline in next few years.

Following these discussions, we decided the target sensitivity of the EB resist for NGL mask fabrication as $100 \mu \mathrm{C} / \mathrm{cm}^{2}$ on $50 \mathrm{kV}$ EB.

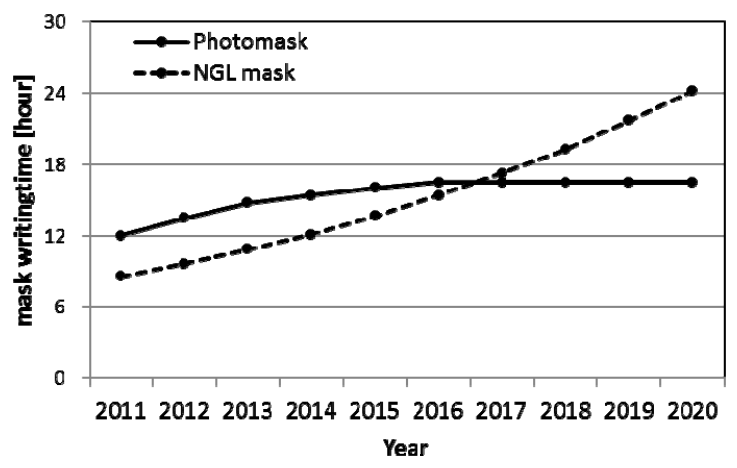

Fig.2. Estimation of writing time of a mask

* mask pattern data is estimated from ITRS2012

**other parameters are estimated from common information

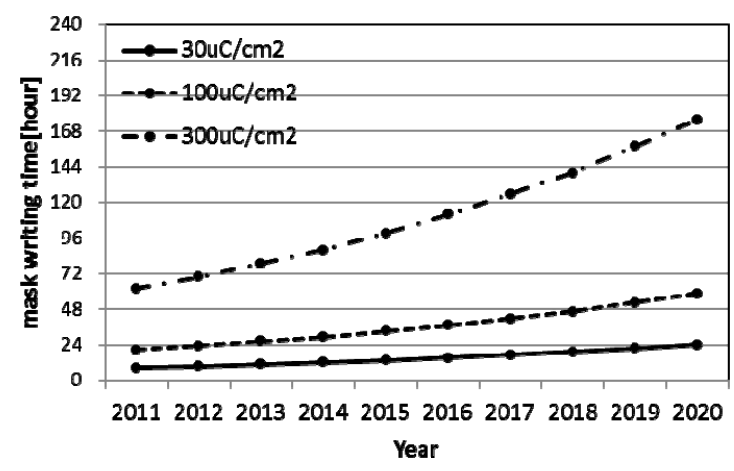

Fig.3. Estimation of writing time of a NGL mask depends on resist sensitivity 
2.2. Benchmark of mask manufacturing process

To examine the performance of today's mask fabrication process, the resolution limit of conventional mask fabricating process was observed experimentally.

A posi-type CAR which is commonly used in photomask fabrication process was coated on mask blanks with $100 \mathrm{~nm}$ thickness. And the line and space patterns were drawn within large area by EBM-8000 [3] (Nuflare Technology, 50kV and VSB type) which is commonly used for advanced mask fabrication. (Here, "large area" is over $100 \mu$ $\mathrm{m}$ area, it means that patterns on center of large area suffer proximity effect uniformly.) After that, the blanks was baked and developed with standard recipe of mask fabrication.

The cross section profile of resist patterns is showed in Fig.4. The optimal exposure dosage was $25 \mu \mathrm{C} / \mathrm{cm}^{2}$ at that time. Down to hp30nm, the resist structures are separated each other. But the pattern collapse starts after resist aspect ratio reached 2. In hp30nm patterns, the collapse appeared over whole lines, so it's hard to judge the resolution limit clearly.

To prevent the resist collapse, the initial resist thickness was reduced to $30 \mathrm{~nm}$. The resist aspect ratio is kept below 2 even in $15 \mathrm{~nm}$ pattern with this thickness.

The results of the experiments with thinner resist thickness are showed in Fig.5. The resist profile is degrading in thinner resist. The line edge roughness becomes worse, and the residual resist pattern height is obviously lower than initial resist thickness. Furthermore, the optimal exposure dosage largely changes from $25 \mu \mathrm{C} / \mathrm{cm}^{2}$ to $38 \mu$ $\mathrm{C} / \mathrm{cm}^{2}$. We deduce these degrading are mainly caused by two reasons. The first reason is the distribution of resist components existing into depth direction, and second one is some kinds of chemical reaction appearing between under layer and resist.

Ignoring the profile degradation, concave and convex shapes are still remaining in below $20 \mathrm{~nm}$ patterns. It shows the possibility to resolve below $20 \mathrm{~nm}$ patterns. But the residual resist height is too low to use as mask material for etching an under layer.

From the result of these experiments, we got the conclusion that today's conventional EB resist don't have enough performance to fabricate NGL mask following with the scaling roadmap indicated in ITRS2012. The challenges for EB resist are keeping residual resist height without pattern collapse, and improving resist profile (roughness etc.) while enhancing pattern resolution.

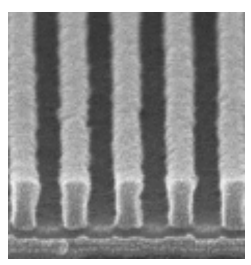

hp50nm

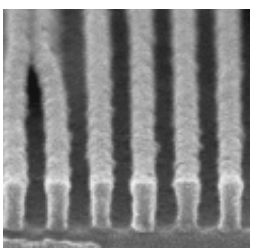

hp40nm

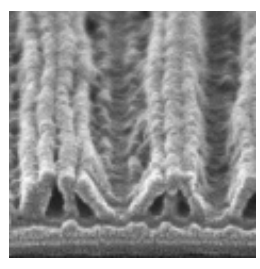

$\mathrm{hp} 30 \mathrm{~nm}$
Fig.4. Cross section profile of conventional resist pattern (100 $\mu \mathrm{m}$ thick.)

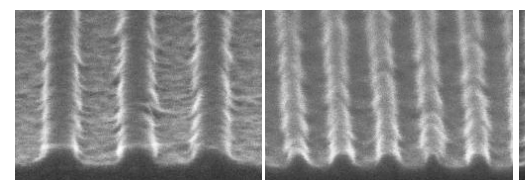

hp50nm

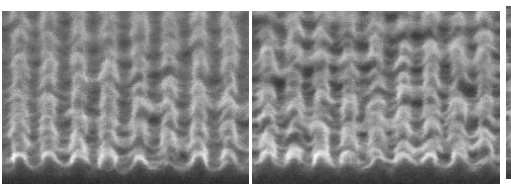

hp20nm hp18nm

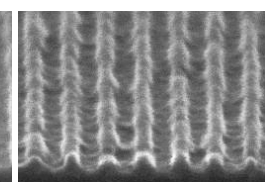

hp24nm
Fig.5. Cross section profile of conventional resist pattern (30 $\mu$ m thick.)

\section{Improvement of pattern resolution}

To catch up the roadmap of mask pattern size scaling, high resolution EB resist has been developing. The target of resist sensitivity is set at $100 \mu \mathrm{C} / \mathrm{cm}^{2}$ on $50 \mathrm{kV}$ EB following former discussion. To achieve this sensitivity, the posi-type CAR has selected as motif material. The resist pattern profiles (defects and collapse) are picked up as metric for evaluating a resist.

\subsection{Improvement of resist resolution}

"Resist A" is finer sample developed until today. But the sensitivity of Resist A is $250 \mu$ $\mathrm{C} / \mathrm{cm}^{2}$ on $50 \mathrm{kV}$ at $50 \%$ pattern coverage. The sensitivity doesn't reach our target described before, 
but we examined its performances as today's best resist sample.

Resolution limit of Resist A was evaluated with same procedure. Basic information of this experiment is showed in Table.1.

Table.1 Experiment conditions

\begin{tabular}{|ll|}
\hline Equipments & $\begin{array}{l}\text { Thin Cr hard mask on 6025 } \\
\text { mask blank }\end{array}$ \\
\hline Resist & $\begin{array}{l}\text { Sample A (posi-type CAR) } \\
\text { 30nm th. }\end{array}$ \\
\hline Writer & $\begin{array}{l}\text { EBM-8000 (50kV/VSB) } \\
\text { *Nuflare Technology }\end{array}$ \\
\hline PEB & $\begin{array}{l}\text { 20min with recommended } \\
\text { temperature }\end{array}$ \\
\hline Develop & TMAH with standard recipe \\
\hline Observation & SEM \\
\hline
\end{tabular}

Fig.6 shows the top-down profile of the line and space pattern formed by Resist A. Down to hp $18 \mathrm{~nm}$ patterns are clearly separated each other, and defects starts appearing in hp16nm patterns. Fig.7 shows the cross section profile of same sample. Lots of defect caused by touched pattern and undissolved resist are observed in hp16nm pattern. These defects are hard to be vanished by controlling the process conditions. PAB and PEB process conditions and development process conditions are optimized before the experiments. If higher exposure dosage is applied for expecting dissolve the residual resist, whole patterns are dissolved by over exposure.

We got conclusion that the resolution limit of Resist A is hp18nm in large area line and space pattern with current mask fabricating equipment.

\subsection{Resist damage by proximity effect}

The proximity effect is specific phenomena appeared in EB lithography. The back scattered electrons from substrate contribute to resist exposure. The resist patterns which aren't exposed by drawing beams directory are exposed by back scattered electrons. The contrast of exposure energy profile is lowered by this phenomenon. So resist pattern profile after developed is degraded.
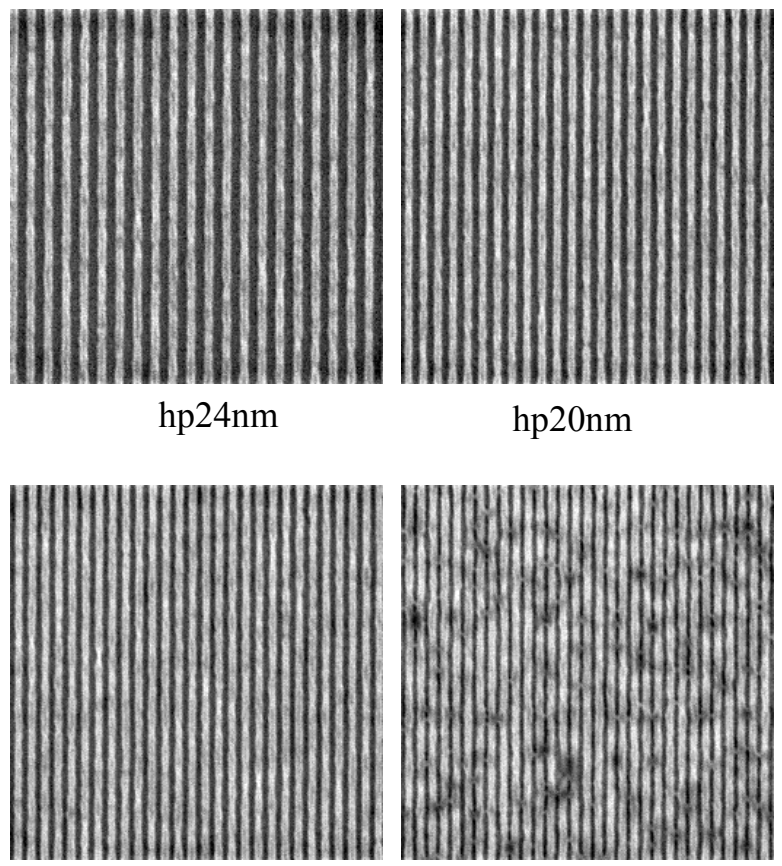

$\mathrm{hp} 18 \mathrm{~nm}$

hp16nm

Fig.6 Top view profile of large area line and space resist pattern (Resist A)

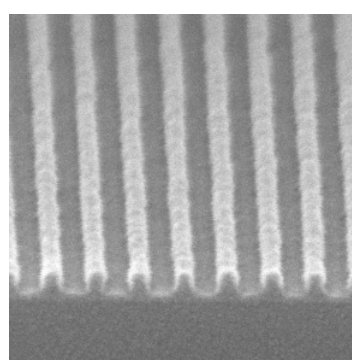

hp24nm

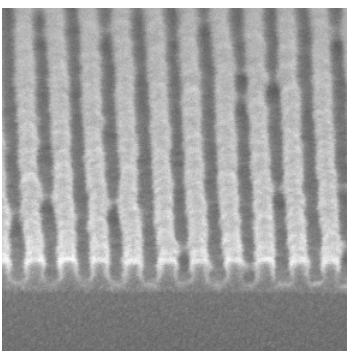

hp18nm

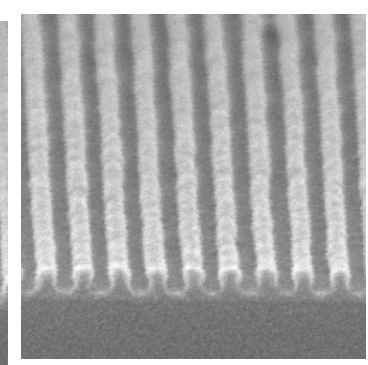

hp20nm

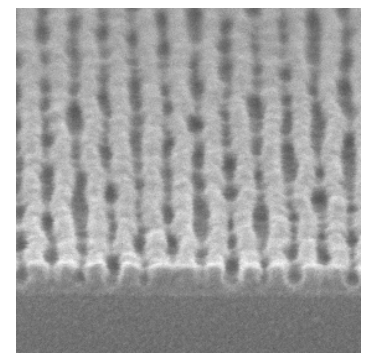

hp16nm
Fig.7. Cross section profile of large area line and space resist pattern (Resist A) 
To examine the impact of proximity effect, small area line and space pattern sample (low pattern density) was prepared. In small area line and space pattern, only 10 lines and spaces exist within back scattering radii. So the amount of back scattered electrons on observed pattern becomes negligibly small. Other hand in large area line and space pattern, whole area within back scattering radii is covered with lines and spaces pattern, so observed pattern suffers the effect of back scatter electrons largely.

Fig.8 shows the cross section profiles of small area line and space pattern sample. The defects in hp16nm patterns are clearly decreased. And the resolution limit of small area line and space pattern is improved from hp18nm to hp16nm. These differences show the damage induced from proximity effect.

In the actual mask patterns used for semiconductor device manufacturing, there exists variety of pattern kinds. Each pattern kinds have different pattern coverage, so the impact of proximity effect differ each other. That means the quality of mask pattern differs depending on pattern kinds, because the damage induced from proximity effect differs each other.

From these experimental results and

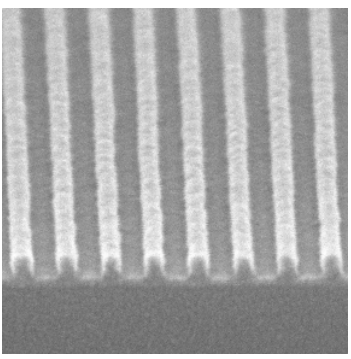

hp24nm

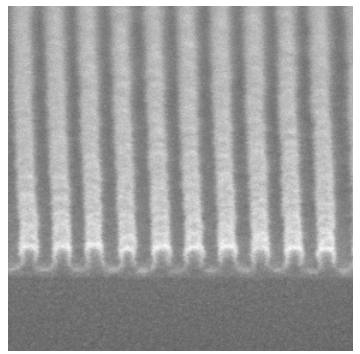

hp18nm

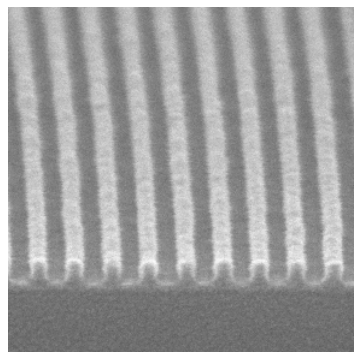

hp20nm

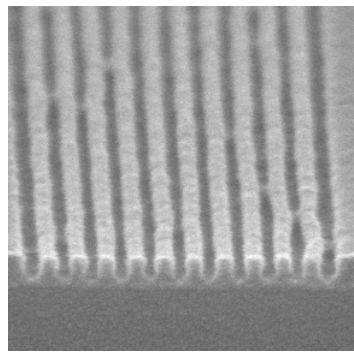

hp16nm
Fig.8. Cross section profile of small area line and space resist pattern (Resist A) discussion, we got the conclusion that the development of EB resist must be progressed with caring the damage induced from the proximity effect. Comparing the results between large area and small area sample is very important procedure.

\subsection{Improvement of beam resolution}

We are trying to improve electron beam resolution too. As described in former section, productivity is major challenges in mask manufacturing. So beam resolution must be improved while following the mask writing time guideline. EBM-9000 is advanced mask writer, newly released in 2013 from Nuflare Technology, Inc. for hp16nm generation's mask writing. This EB writer has performance of higher throughput which meets the next generation mask manufacturing.

We examined the pattern resolution with EBM-9000.Experimental conditions are same in Table. 1 except EB writer.

The results with large area line and space pattern are showed in Fig.9. In the top view SEM image, hp16nm patterns are clearly separated each other. The resolution limit has improved about $2 \mathrm{~nm}$ by the improvement of EB writer.

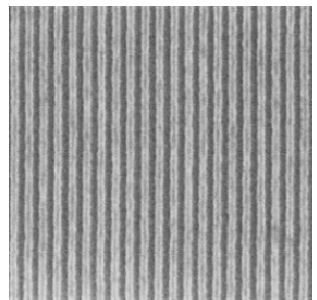

hp24nm

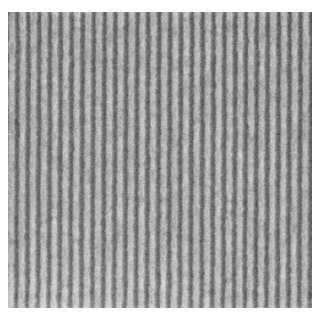

hp18nm

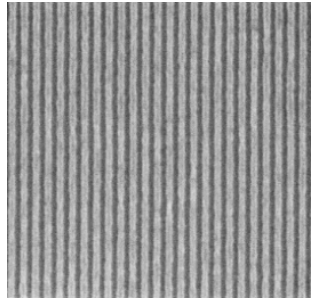

hp20nm

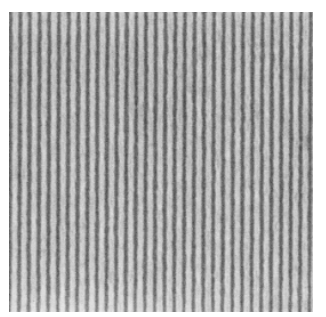

hp16nm
Fig.9. Cross section profile of large area line and space resist pattern (Reisist A combined with new EB writer) 


\section{Conclusion}

According to the scaling roadmap indicated in ITRS, the pattern size on NGL mask shrinks to below 20nm in 2017, after that it goes to around 10 $\mathrm{nm}$ by 2020 . Following this roadmap, improving EB resist and EB writer is important challenge.

Considering the productivity of mask manufacturing, around $100 \mu \mathrm{C} / \mathrm{cm}^{2}$ on $50 \mathrm{kV}$ EB is expected as sensitivity of EB resist for NGL mask fabrication. CAR is suitable to follow this guideline because of its high sensitivity. But conventional CAR for EB doesn't have enough performance in pattern resolution.

Advanced CAR was developed for improving the pattern resolution, and its resolution limit combined with conventional mask fabricating equipment reached to $\mathrm{hp} 18 \mathrm{~nm}$. By using advanced EB writer, further enhancement of the resolution limits up to hp16nm has been expected.

The resist profile damage induced by proximity effect was investigated. The resolution limit was degraded about $2 \mathrm{~nm}$ by the impact of proximity effect. It is a fatal damage for fabricating practical NGL mask. This result indicates that the pattern quality will be varied depending on pattern kinds included in a mask.

\section{Acknowledgements}

The authors acknowledge H. Anze and T. Katsumata Nuflare Technology, Inc. for supporting this work.

\section{References}

1. ITRS. "International Technology Roadmap for semiconductors (ITRS 2012 Update)". http://www. itrs.net

2. H. Sunaoshi, Y. Tachikawa, H. Higurashi, T. Iijima, J. Suzuki, T. Kamikubo, K. Ohtoshi, H. Anze, T. Katsumata, N. Nakayamada, S. Hara, S. Tamamushi and Y. Ogawa, Proc. SPIE, 6283 (2006) 628306.

3. S. Yoshitake, T. Kamikubo, N. Nakayamada, K. Hattori, H. Ando, T. Iijima, K.Ohtoshi, K. Saito, R. Yoshikawa, S. Tamamushi, R. Tomiyoshi, H. Higurashi, Y. Hattori, S. Tsuchiya, M. Katoh, K. Suzuki, Y. Tachikawa, M. Ogasawara, V. Katsap, S. D. Golladay, R.A. Kendall, Proc. SPIE, 8166 (2011) 81661D. 